\title{
Clustering-Based Relational Unsupervised Representation Learning with an Explicit Distributed Representation
}

\author{
Sebastijan Dumančić and Hendrik Blockeel \\ Computer Science Department, KU Leuven, Belgium \\ \{sebastijan.dumancic,hendrik.blockeel\}@ cs.kuleuven.be
}

\begin{abstract}
The goal of unsupervised representation learning is to extract a new representation of data, such that solving many different tasks becomes easier. Existing methods typically focus on vectorized data and offer little support for relational data, which additionally describe relationships among instances. In this work we introduce an approach for relational unsupervised representation learning. Viewing a relational dataset as a hypergraph, new features are obtained by clustering vertices and hyperedges. To find a representation suited for many relational learning tasks, a wide range of similarities between relational objects is considered, e.g. feature and structural similarities. We experimentally evaluate the proposed approach and show that models learned on such latent representations perform better, have lower complexity, and outperform the existing approaches on classification tasks.
\end{abstract}

\section{Introduction}

Every machine learning task inherently depends on the quality of provided features. A good set of features is thus a crucial precondition for the good performance of any classifier. Yet, finding such a set in practice has proven to be a tedious and time-consuming task. Furthermore, substantial domain knowledge and exploration are often required. To address this issue, representation learning [Bengio, 2009] focuses on automatic learning of good multi-level data representations.

One of the prominent paradigms within representation learning, and the one this work focuses on, is unsupervised representation learning $(\mathcal{U R} \mathcal{L})$ [Hinton and Salakhutdinov, 2006; Bengio et al., 2007; Ranzato et al., 2007]. These approaches take a generative stance on learning a feature hierarchy and require no supervision. As supervision is ignored, the obtained representation are not tailored for one specific task but can rather be shared among multiple tasks. Intuitively, these methods find useful features by compressing the original data by means of a multiple-clustering procedure, in which an instance can belong to more than one cluster. The obtained features indicate cluster assignments of instances and, thus, a classifier learns from cluster memberships instead of the original data.
One major limitation of the existing approaches is that they focus on vectorized data representations. Hence, the ubiquitous and abundant structured and relational data, which additionally describes relationships between instances, are not well supported. This data is often viewed as a hypergraph ${ }^{1}$, in which instances form vertices and their relationships form hyperedges. Such data emerges in many real-life problems. For instance, chemical and biological data describing molecules or protein interaction networks are naturally expressed in graphstructured formats. In social networks, many instances interact with each other. Relational data is commonly expressed in predicate logic, a powerful language unifying the representation of vectors, graphs and sequences. This, consequently, subsumes any data stored in a relational database.

Here we focus on the problem of unsupervised learning a feature hierarchy with relational data. To this end, we introduce CUR ${ }^{2}$ LED - a clustering-based unsupervised relational representation learning with explicit distributed representation. CUR ${ }^{2}$ LED is inspired by the work of Coates et al. (2011) in which the authors introduce a general pipeline for learning a feature hierarchy by means of clustering. Assuming a spatial order of features (i.e., pixels), the introduced framework (i) extracts image patches, i.e., subsets of pixel from the original images candidating as a potential high-level feature, (ii) pre-processes each patch (e.g. normalization), and (iii) learns a feature-mapping by clustering image patches. The authors show that such general procedure with a simple k-means algorithm can perform as well as the specialized algorithms, such as auto-encoders and Restricted Boltzmann machines.

CUR ${ }^{2}$ LED learns a new representation by clustering both instances and their relationships. A distinctive feature of $\mathrm{CUR}^{2} \mathrm{LED}$ is that the relational structure is preserved throughout the hierarchy, contrasted to the existing approaches that replace relational structures with vectors. Another distinctive feature is the notion of similarity interpretation. When clustering relational data, a similarity of relational objects is an ambiguous concept. Two relational objects might be similar according to their attributes, relationships, or a combination of both. The notion of similarity interpretation precisely states the exact source of similarity used.

CUR ${ }^{2}$ LED exploits this ambiguity to its advantage by using

\footnotetext{
${ }^{1}$ A hypergraph is a graph in which edges can connect more than two vertices.
} 
the similarity interpretations to encode a distributed representation of data - one of the pillars underlying the success of representation learning methods. Intuitively, it refers to a concept of reasonably-sized representation that captures a huge number of possible configurations [Bengio et al., 2013]. In contrast to the one-hot representations which require $N$ parameters to represent $N$ regions, distributed representations require $N$ parameters to represent up to $2^{N}$ regions. The main difference is that a concept within a distributed representation is represented with several independently manipulated factors, instead of exactly one factor as with one-hot representations. Thus, such representations are substantially more expressive. The similarity interpretation defines the exact factors that can be manipulated individually to represent individual concepts.

The contributions of this paper include (i) a general framework for learning relational feature hierarchies by means of clustering, (ii) a principled way of generating distributed relational representations based on different similarity interpretations, (iii) a general framework for hyperedge clustering and (iv) the experimental evaluation of the proposed framework.

In the following section, we briefly review related work. Next, we outline our approach and discuss arising issues in Section 3 . We then briefly present the similarity measure used for clustering relational data, discuss its extension towards hyperedge clustering, and formally define the notion of similarity interpretation. Experimental results are discussed in Section 5 .

\section{Related Work}

Clustering has been previously recognized as an effective way of enhancing relational learners. Popescul and Ungar (2004) apply k-means clustering to instances, create predicates for new clusters and add them to the original data. Multiple relational clustering (MRC) [Kok and Domingos, 2007; 2008] is a relational probabilistic clustering framework based on Markov logic networks [Richardson and Domingos, 2006] clustering both vertices and relationships. Both approaches are instances of predicate invention [Kramer, 1995; Craven and Slattery, 2001], concerned with extending the vocabulary given to a learner by discovering novel concepts in data. CUR ${ }^{2}$ LED differs in several ways. Whereas Popescul and Ungar develop a method specifically for document classification, CUR ${ }^{2}$ LED is a general off-the-shelf procedure that can be applied to any relational domain. Moreover, CUR ${ }^{2}$ LED clusters both instances and relations, whereas Popescul and Ungar cluster only instances. In contrast to MRC which does not put any assumptions in the model, CUR ${ }^{2}$ LED is a more informed approach that explicitly defines different notions of relational similarity to be used for clustering. Though MRC was used as a component in structure learning, it does not provide new language constructs, but simplifies the search over possible formulas. CUR ${ }^{2}$ LED learns a model directly from the new features.

A related problem is community detection [Karypis and Kumar, 1998; Fortunato, 2010] concerned with identifying a densely connected components in graphs. CUR ${ }^{2}$ LED considers a more general problem in which disconnected vertices (and edges) can form clusters as well.
Much of the recent work focused on embedding relational data into vector spaces [Nickel et al., 2016; Niepert, 2016; Bordes et al., 2011; 2013; Niepert et al., 2016]. The essential idea behind these approaches is to map relational concepts to a low-dimensional vectors spaces, and replace logical reasoning with algebra. Rather than inventing an Euclidean space of relational instances, $\mathrm{CUR}^{2} \mathrm{LED}$ relies on clustering and a variety of similarity measures to create new features.

\section{Representation Learning via Clustering}

The complexity of relational data causes several issues in devising a general relational feature hierarchy: (1) what should be clustered, (2) how to estimate a similarity in relational data, and (3) how to choose the structure of a feature hierarchy?

(1) In the i.i.d. case (drawn independently from the same population), the dataset contains only instances and their features, thus, one clusters the instances. However, relational data additionally describes relationships among instances and varies from a single large network of many interconnected entities (a mega-example) to a set of many disconnected networks where each network is an example. Ideally, one would address both cases. CUR ${ }^{2}$ LED assumes that relational data is provided as a labelled hypergraph, where examples form vertices and relations between them form hyperedges, and does not make a distinction between the above-mentioned cases. Formally said, the data structure is a typed, labelled hypergraph $H=(V, E, \tau, \lambda)$ with $V$ being a set of vertices, $E$ a set of hyperedges, $\tau$ a function assigning a type to each vertex and hyperedge, and $\lambda$ a function assigning a vector of values to each vertex. CUR ${ }^{2}$ LED learns a new representation by clustering both vertices and hyperedges in a hypergraph. Considering that vertices have associated types, CUR ${ }^{2}$ LED does not allow mixing of types, i.e., a cluster can contain only vertices of the same type. The same holds for hyperedges which connect vertices of different types.

(2) The features are the only source of similarity between instances in the i.i.d. data. In the relational context, a similarity is an ambiguous notion that can originate in the features of relational objects, structures of their neighbourhoods (both feature- and relationship-wise), interconnectivity or graph proximity, just to name a few. Furthermore, which interpretation is needed for a particular task is not known in advance, making $\mathcal{U} \mathcal{R} \mathcal{L}$ inherently more difficult. To find a representation effective for many tasks, $\mathrm{CUR}^{2} \mathrm{LED}$ addresses multiple interpretations of relational similarity simultaneously. How exactly that is achieved is discussed in the next section, together with a similarity measure used for this purpose.

(3) Defining a feature hierarchy requires the specification of the number of layers and the number of hidden features (i.e., clusters) within each layer. How to automatically construct such hierarchies is currently under-explored. Consequently, the performance of these methods is sensitive to the parameter setting, requiring substantial expertise in order to choose the optimal number of features. This constitutes a major bottleneck for relational type-aware feature hierarchies, as separate values should be chosen for each type in data (and combination thereof for hyperedges).

To tackle this infeasibility, CUR $^{2}$ LED builds upon a vast 


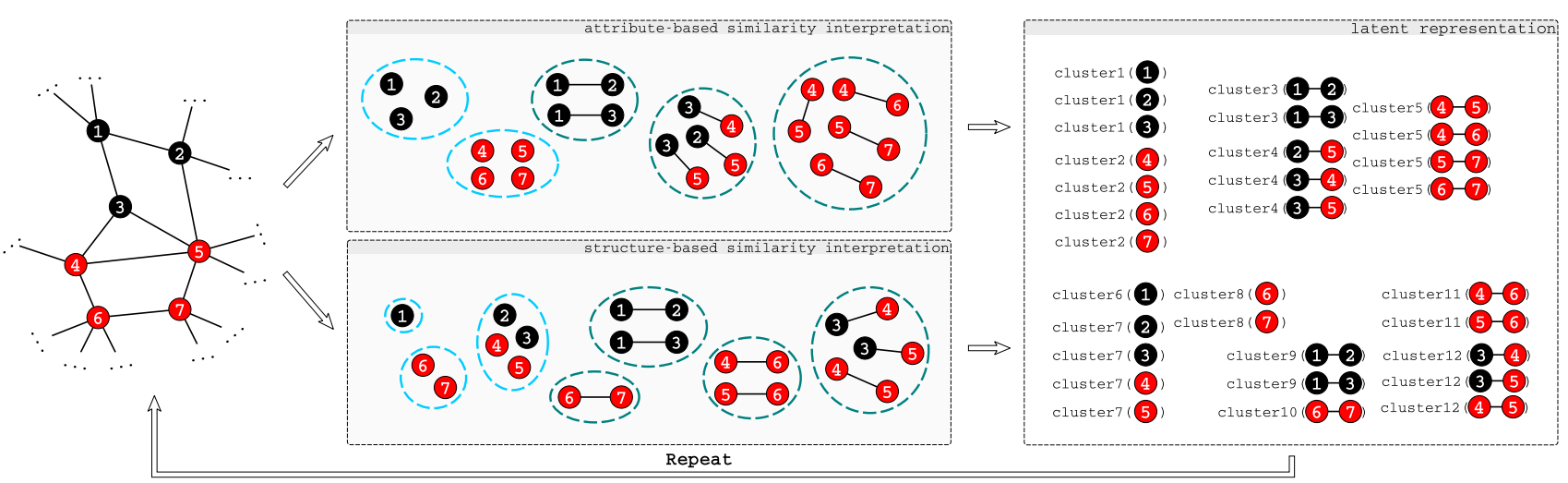

Figure 1: An illustration of CUR ${ }^{2}$ LED procedure. The left-most figure represents a given hypergraph, where colour of a vertex indicates its feature value. The graph (i.e., vertices and edges) is then clustered according to different similarity interpretations. The upper clustering is based on vertex attributes: the vertices are clustered into red and black ones, while the edges are clustered according to the colour of the vertices they connect. The bottom clustering is based on the structure of the neighbourhoods. The vertices are clustered into a group that have only black neighbours $(\{1\})$, only red neighbours $(\{6,7\})$, and neighbours of both colours $(\{2,3,4,5\})$. The edges are clustered into a group of edges connecting black vertices with only black neighbours and black vertices with red neighbours $(\{1-2,1-3\})$, a group of edges connecting red vertices with only red neighbours to red vertices with neighbours of both colour $(\{6-7\})$, and so on. The final step transforms the obtained clusterings into a relational representation. The procedure can further be repeated to create more layers of features.

literature on the clustering selection problem [Arbelaitz et al., 2013], which is concerned with the selection of optimal number of clusters from data. This automatic clustering selection strategies mitigate the problem of the manual specification of feature hierarchies. CUR ${ }^{2}$ LED leverages two distinct approaches: (1) a difference-like criterion [Vendramin et al., 2010], and (2) a quality based criterion of Silhouette index [Rousseeuw, 1987].

Difference-like criteria assess relative improvements on some relevant characteristic of the data (e.g. within-cluster similarity) over a set of successive data partitions produced by gradually increasing the number of clusters $(N)$. It attempts to identify a prominent knee - a point when the given quality measure saturates and the further increase of $N$ can offer only marginal benefit. Following the suggestion in [Vendramin et al., 2010], we choose the number of clusters as the one that achieves the highest value of the following formula:

$$
D(k)=\left|\frac{C(k-1)-C(k)}{C(k)-C(k+1)}\right|-\alpha \cdot k
$$

where $C(k)$ is the intra-cluster similarity, $k$ is the number of clusters and $\alpha$ a user-specified penalty on the number of clusters.

The Silhouette index evaluates a cohesion, i.e., how similar an instance is to its own cluster, and a separation, i.e., how similar an instance is to the other clusters. It is defined as:

$$
S(i)=\frac{a(i)-b(i)}{\max \{a(i), b(i)\}}
$$

where $i$ is an instance, $a(i)$ is an average dissimilarity of $i$ to the rest of the instances in the same cluster, and $b(i)$ the lowest dissimilarity of $i$ to any other cluster. Higher values indicate a better fit of the data.

$\mathcal{C}$-representation. Once the clusters are obtained, we will represent them in the following form. For each clus- ter of vertices we create a unary predicate in the form of clusterID(vertex) where vertex is an identifier of a specific vertex. Similarly, for each cluster of hyperedges we create a $n$-ary predicate in the form of clusterID (vertex $1, \ldots$, vertex ${ }_{n}$ ), which takes an ordered set of $n$ vertices as arguments. Truth instantiations of defined predicates reflect cluster memberships. We refer to the cluster-induced representation as a $\mathcal{C}$-representation.

The introduced pipeline is illustrated in Figure 1 . CUR $^{2}$ LED specified thus far describes a meta-procedure how to use any clustering algorithm to obtain a latent representation. In the experiments we use spectral and hierarchical clustering.

\section{Similarity of Relational Structures}

CUR ${ }^{2}$ LED relies on ReCeNT [Dumančić and Blockeel, 2017], a relational clustering framework focused on clustering vertices in a hypergraph. What makes ReCeNT an attractive relational clustering framework is the wide range of similarities it considers. Furthermore, which similarity is used is easily adaptable with just a few parameters. We provide a concise and intuitive description here, and refer the reader to the original paper for the details.

The core concept of ReCeNT is a neighbourhood tree (NT). The NT is a rooted directed graph describing a neighbourhood of a certain vertex in the hypergraph. It provides a summary of all paths that can be taken, starting from that particular vertex. The depth of a NT, i.e., how many vertices a path can contain excluding the root vertex, is pre-specified. ReCeNT compares two vertices by comparing their NTs.

This comparison is achieved by first decomposing the NT into different multisets. The multiset $V_{t}^{l}(g)$ contains all vertices of type $t$ at distance $l$ of a particular NT $g . E^{l}(g)$ is the multiset of hyperedge labels between vertices of distances $l$ and $l+1$. Finally, $B_{t, a}^{l}(g)$ is the multiset of values of attribute 
$a$ observed among the nodes of type $t$ at distance $l$. Using only these three types of multisets, one can express a wide range of similarities. What ReCeNT considers are:

1. the similarity of the root vertices in terms of attribute values, by means of $B_{t, a}^{0}$

2. the similarity of attribute values of the neighbouring vertices, by means of $B_{t, a}^{l>0}$

3. the connectivity of the root vertices, by means of $V_{t}^{l}$

4. the similarity of neighbourhoods in terms of the vertex identities, by means of $V_{t}^{l}$

5. the similarity of hyperedge labels of two neighbourhoods, by means of $E^{l}$.

Each of the components represents a distinct notion of similarity. We will refer to them as core similarities. These core similarities can further be combined to represent more complex similarities.

\subsection{Hyperedge Similarity}

In its original form, ReCeNT clusters vertices in a hypergraph. To support hyperedge clustering with CUR $^{2}$ LED, we introduce a general framework for hyperedge similarity. It views hyperedges as ordered sets of vertices, and thus ordered sets of NTs.

Let $\mathcal{N}$ be a set of NTs. Let $\Theta$ denote summary operations on sets of values such as mean, minimum and maximum. Let $\Lambda$ denote set operators such as union and intersection. Let $f$ : $\mathcal{N}^{2} \rightarrow \mathbb{R}$ be a similarity between two NTs, e.g. the similarity measure introduced by ReCeNT. The framework introduces two types of hyperedge similarity, namely combination and merging.

Definition 1. A combination similarity is a function $c$ : $\mathcal{N}^{n} \times \mathcal{N}^{n} \times \Theta \rightarrow \mathbb{R}$ which compares two hyperedges, $e_{1}=$ $\left(v_{1}^{1}, \ldots, v_{1}^{n}\right)$ and $e_{1}=\left(v_{2}^{1}, \ldots, v_{2}^{n}\right)$, by comparing the individual NTs respecting the order, $s=\left(f\left(v_{1}^{1}, v_{2}^{1}\right), \ldots, f\left(v_{1}^{i}, v_{2}^{i}\right)\right)$, and summarizing respective similarities with $\theta \in \Theta, \theta(s)$.

Definition 2. A merging similarity is a function $m: 2^{\mathcal{N}} \times$ $2^{\mathcal{N}} \times \Lambda \rightarrow \mathbb{R}$ which compares two hyperedges, $e_{1}=$ $\left(v_{1}^{1}, \ldots, v_{1}^{n}\right)$ and $e_{1}=\left(v_{2}^{1}, \ldots, v_{2}^{n}\right)$, by first merging the NTs within a hyperedge with merging operator $\lambda \in \Lambda, s_{1}=$ $\lambda\left(v_{1}^{1}, \ldots, v_{1}^{i}\right)$ and $s_{2}=\lambda\left(v_{2}^{1}, \ldots, v_{2}^{i}\right)$ and comparing the resulting NTs, $f\left(s_{1}, s_{2}\right)$.

Merging two NTs involves merging their respecting multisets with a merging operator $\lambda$, respecting the level. For instance, consider set union as $\lambda$, and $g^{\prime}$ and $g^{\prime \prime}$ as the NTs to be merged. Then, merging the multisets $V_{t}^{l}\left(g^{\prime}\right)$ and $V_{t}^{l}\left(g^{\prime \prime}\right)$ results in a multiset $V_{t}^{l}\left(\lambda\left(g^{\prime}, g^{\prime \prime}\right)\right)=V_{t}^{l}\left(g^{\prime}\right) \cup V_{t}^{l}\left(g^{\prime \prime}\right)$.

Both formulations reduce the problem to the comparison of NTs, but offer alternative views. While merging ignores the order of vertices in a hyperedge, combination respects it. Accordingly, merging describes the neighbourhood of a hyperedge, while combination examines the similarity of vertices participating in a hyperedge. In this work we use union as the merging operator, and mean as the combination operator.

\subsection{Similarity Interpretation}

Finally, we formally introduce the notion of similarity interpretation.

Definition 3. Let $\left(w_{1}, w_{2}, w_{3}, w_{4}, w_{5}\right)$ be the weights associated with the core similarities. A similarity interpretation is the value assignments to the weights $\left(w_{1}, w_{2}, w_{3}, w_{4}, w_{5}\right)$.

Thus, it allows us to precisely control aspects of similarity considered for representation learning. For example, setting $w_{1}=1, w_{2,3,4,5}=0$ uses only the attributes of vertices for comparison. Setting $w_{3}=1, w_{1,2,4,5}=0$ on the other hand would identify clusters as a densely connected components. As the similarity interpretation is provided by the user, we say it explicitly defines the distributed representation.

\section{Experiments and Results}

Datasets. We have used the following 6 datasets to evaluate the potential of this approach. The IMDB dataset describes a set of movies with people acting in or directing them. The UW-CSE dataset describes the interactions of employees at the University of Washington and their roles, publications and the courses they teach. The Mutagenesis dataset describes chemical compounds and atoms they consist of. The WebKB dataset consists of pages and links collected from the Cornell University's web page. The Terrorists dataset describes terrorist attacks each assigned one of 6 labels indicating the type of the attack. The Hepatitis dataset describes a set of patients with hepatitis types B and C.

Evaluation procedure. In principle, a latent representation should make learning easier by capturing complex dependencies in data more explicitly. Though that is difficult to formalize, a consequence should be that a model learned on the latent representation is (i) less complex, and (ii) possibly performs better. To verify whether that is the case with the representation created by $\mathrm{CUR}^{2} \mathrm{LED}$, we answer the following questions:

(Q1) do representations learned by $C U R^{2} L E D$ induce models of lower complexity compared to the ones induced on the original representation?

(Q2) if the original data representation is sufficient to solve a task efficiently, does $\mathcal{C}$-representation preserves the relevant information?

(Q3) if the original data representation is not sufficient to solve the task, does a $\mathcal{C}$-representation improve the performance of a relational classifier?

(Q4) can the appropriate parameters for a specific dataset be found by the model selection?

(Q5) how does CUR ${ }^{2} L E D$ compare to MRC, which is the closest related work?

In order to do so, we use TILDE [Blockeel and De Raedt, 1998], a relational decision tree learner, and perform 5 -fold cross validation. $\mathcal{C}$-representations and TILDE were learned on training folds, and the objects from the test fold were mapped to the $\mathcal{C}$-representation and used to test TILDE. The following similarity interpretation were used for each dataset: $(0.5,0.5,0.0,0.0,0.0),(0.0,0.0,0.33,0.33,0.34)$, 
Table 1: Performance comparison for TILDE models learned on the original and $\mathcal{C}$-representations. The first column specifies the parameters used for $\mathcal{C}$-representation, i.e., clustering algorithm (S-spectral, H-hierarchical), selection criterion and its parameter values. Both accuracies on a test set (Acc) and complexities (Cplx) are reported.

\begin{tabular}{|c|c|c|c|c|c|c|c|c|c|c|c|c|c|}
\hline & \multirow{2}{*}{ Setup } & \multicolumn{2}{|c|}{ IMDB } & \multicolumn{2}{|c|}{ UWCSE } & \multicolumn{2}{|c|}{ Mutagenesis } & \multicolumn{2}{|c|}{ Terrorists } & \multicolumn{2}{|c|}{ Hepatitis } & \multicolumn{2}{|c|}{ WebKB } \\
\hline & & Acc & Cplx & Acc & Cplx & Acc & Cplx & Acc & Cplx & Acc & Cplx & Acc & Cplx \\
\hline & Original & 1.0 & 2.0 & 0.99 & 3.0 & 0.76 & 27.2 & 0.72 & 86.4 & 0.81 & 22.4 & 0.81 & 18.2 \\
\hline \multirow{8}{*}{ 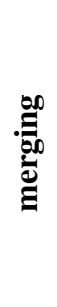 } & $\mathrm{S}, \alpha=0.01$ & 1.0 & 1.0 & 0.99 & 1.2 & 0.79 & 6.6 & 0.71 & 34.4 & 0.86 & 19.66 & 0.89 & 13.6 \\
\hline & $\mathrm{S}, \alpha=0.05$ & 1.0 & 1.0 & 0.99 & 1.0 & 0.78 & 2. & 0.65 & 21.6 & 0.90 & 7.6 & 0.85 & 15.6 \\
\hline & $\mathrm{S}, \alpha=0.1$ & 1.0 & 1.0 & 0.99 & 1.2 & 0.78 & 1.8 & 0.66 & 32.4 & 0.90 & 6.5 & 0.87 & 17.8 \\
\hline & S, silhouette & 1.0 & 1.0 & 0.99 & 1.0 & 0.78 & 2.0 & 0.6 & 23.6 & 0.93 & 5.33 & 0.87 & 14.8 \\
\hline & $\mathrm{H}, \alpha=0.01$ & 1.0 & 1.0 & 0.98 & 4.4 & 0.83 & 2.0 & 0.48 & 9.4 & 0.86 & 12.0 & 0.83 & 12.6 \\
\hline & $\mathrm{H}, \alpha=0.05$ & 1.0 & 1.0 & 0.99 & 4.2 & & 2. & & 11.6 & 0.82 & 16.0 & 0.69 & 27.2 \\
\hline & $\mathrm{H}, \alpha$ & 1.0 & 1.0 & 0.99 & 4.0 & 0.79 & 5.2 & 0.47 & 8.8 & 0.82 & 13.4 & 0.61 & 32.2 \\
\hline & H,silhouette & 1.0 & 1.0 & 0.98 & 1.0 & 0.80 & 3.4 & 0.47 & 13.0 & 0.93 & 8.66 & 0.68 & 18.0 \\
\hline \multirow{8}{*}{ 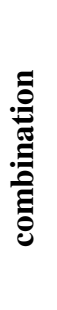 } & $\mathrm{S}, \alpha=0.01$ & 1.0 & 10 & 0.99 & 12 & 079 & 2.0 & 0.72 & 24.0 & 0.90 & 76 & 0.90 & 11.8 \\
\hline & $\mathrm{S}, \alpha=$ & 1.0 & 1. & 0.99 & 1. & 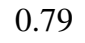 & 2. & 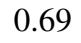 & 22.8 & o & 12.2 & 0.86 & 10.0 \\
\hline & $\mathrm{S}, \alpha=0.1$ & 1.0 & 1.0 & 1.0 & 1.1 & 0.76 & 2. & 0.66 & 16.8 & 0.90 & 12.6 & 0.87 & 17.0 \\
\hline & S,silhouette & 1.0 & 1.0 & 0.99 & 1.0 & 0.77 & 2.0 & 0.6 & 24.2 & 0.93 & 16.4 & 0.88 & 13.8 \\
\hline & $\mathrm{H}, \alpha=0.01$ & 1.0 & 1. & 0.99 & 2. & 0.7 & 4. & 0.51 & 306 & 0.80 & 29.33 & 0.83 & 12.6 \\
\hline & $\mathrm{H}, \alpha=0.05$ & 1.0 & 1.0 & 0.99 & 2.8 & 0.78 & 2.8 & 0.51 & 30.6 & 0.82 & 16.33 & 0.69 & 27.2 \\
\hline & $\mathrm{H}, \alpha=0.1$ & 1.0 & 1.0 & 0.99 & 2.8 & 0.78 & 11.0 & 0.50 & 27.3 & 0.78 & 14.0 & 0.61 & 32.2 \\
\hline & H,silhouette & 1.0 & 1.0 & 0.99 & 2.0 & 0.80 & 4.0 & 0.50 & 30.0 & 0.83 & 11.6 & 0.68 & 18.0 \\
\hline \multirow{3}{*}{$\stackrel{U}{\Sigma}$} & $\lambda=$ & 18 & 1. & 0.93 & 21.0 & 0.6 & 0 & 064 & 138.7 & 061 & 99.4 & 0.64 & 44 \\
\hline & $\lambda=-5$ & 1.0 & 1.0 & 0.9 & 25 & 0.6 & 23. & 0. & 126.5 & 0.8 & 64.8 & 0.68 & 40.0 \\
\hline & $\lambda=-10$ & 1.0 & 1.0 & 0.96 & 13.7 & 0.72 & 35.0 & 0.51 & 102.1 & 0.57 & 5.7 & 0.66 & 40.8 \\
\hline
\end{tabular}

$(0.2,0.2,0.2,0.2,0.2)$. The first set of weights uses only the attribute information, the second one only the link information, while the last one combines every component.

As a complexity measure of a model we use the number of nodes a trained TILDE model has. We use the following values for the $\alpha$ parameter in Equation 1: $\{0.1,0.05,0.01\}$. In the case of MRC, we used the following values for the $\lambda$ parameter: $\{-1,-5,-10\}$. The $\lambda$ parameter has the same role as $\alpha$ in the proposed approach, affecting the number of clusters chosen for each type ${ }^{2}$.

\section{Results}

To answer the above mentioned questions, we perform two types of experiments. Table 1 summarizes the results of cross validation. The accuracies on test set and the complexities of TILDE models are stated for both original and $\mathcal{C}$-representations. Table 2 summarizes the results of the model selection where we dedicate one fold as a validation set, and perform the cross validation on the remaining folds to identify the best parameter values (i.e., the choice of a clustering algorithm, a clustering selection procedure and the appropriate hyperedge similarity) for each dataset.

Q1. Table 1 shows that the models learned on $\mathfrak{C}$ representation consistently have lower complexity than the ones learned on the original data. That is especially the case when $\mathcal{C}$-representation is obtained by spectral clustering,

\footnotetext{
${ }^{2}$ Note that it is difficult to exactly match the values of $\alpha$ and $\lambda$ as both methods operate on different scales, and the authors do not provide a way how to choose an appropriate value
}

which consistently results in a model of a lower complexity. The reduction of complexity can even be surprisingly substantial, for instance on the Mutagenesis and Hepatitis datasets where the model complexities are reduced by factors of 10 and 4 , respectively. When the $\mathcal{C}$-representation is obtained with hierarchical clustering, models of lower complexity are obtained on all datasets except the WebKB and UWCSE datasets. These results suggest that the $\mathcal{C}$-representation in general makes complex dependencies easier to detect and express.

Q2. The IMDB and UWCSE datasets are considered as easy relational datasets, where the classes are separable by a single attribute or a relationship. Thus, TILDE is able to achieve almost perfect performance with the original data. The original representation is therefore sufficient to solve the task, and we are interested whether the relevant information will be preserved within the $\mathcal{C}$-representation. The results in Table 1 do suggest so, as TILDE achieves identical performance regardless of the representation.

Q3. The remaining datasets are more difficult than the previously discussed ones. On the Mutagenesis, Hepatitis and WebKB datasets, $\mathcal{C}$-representation improves the performance. On the Terrorists dataset, however, no improvement in performance is observed. What distinguishes this dataset from the others is that it contains only two edge types (indicating colocated attack, or ones organized by the same organization), an abundant number of features, while other datasets are substantially more interconnected. Thus, focusing on the relational information is not as beneficial as the features themselves.

These results suggest that $\mathcal{C}$-representations indeed improve 
Table 2: Model selection results. For each dataset, a selected parameters are reported together with the accuracies on the training and test sets. The first element indicates the selected clustering algorithm (S-spectral, H-hierarchical), the second one the clustering selection criteria, while the last one indicates the hyperedge similarity (C-combination, M-merging). The last column indicates the performance on the original data representation.

\begin{tabular}{ccccc}
\hline Dataset & Parameters & Training & Validation & Original \\
\hline IMDB & all & 1.0 & $\mathbf{1 . 0}$ & $\mathbf{1 . 0}$ \\
UWCSE & S, silhouette, C & 0.99 & $\mathbf{1 . 0}$ & 0.99 \\
Mutagenesis & H, $\alpha=0.01$, M & 0.86 & $\mathbf{0 . 8 4}$ & 0.79 \\
Hepatitis & S, silhouette, M & 0.92 & $\mathbf{0 . 8 9}$ & 0.8 \\
WebKB & S, $\alpha=0.01, \mathrm{C}$ & 0.88 & $\mathbf{0 . 8 8}$ & 0.79 \\
Terrorists & S, $\alpha=0.01, \mathrm{C}$ & 0.70 & 0.69 & $\mathbf{0 . 7 1}$ \\
\hline
\end{tabular}

performance of the classifier, compared to the one learned on the original data representation. First, the $\mathcal{C}$-representation created with spectral clustering consistently performs better on all datasets, except the Terrorists one. Second, if the learning task does not have a strong relational component, then $\mathcal{C}$ representations are not beneficial and can even hurt the performance. Third, the choice of a clustering algorithm matters, and spectral clustering does a better job in our experiments - it always results in improved or at least equally good performance. Fourth, the choice of treating hyperedges as ordered (by combination) or unordered (merging) sets is data-dependent, and the difference in performance is observed.

Combining the results from $\mathbf{Q 1}, \mathbf{Q 2}$ and $\mathbf{Q 3}$ shows that the main benefit of $\mathrm{CUR}^{2} \mathrm{LED}$ is the transformation of data such that it becomes easier to express complex dependencies. Consequently, the obtained models have lower complexities and their performance often improves.

Q4. To ensure that the previously discussed results do not over-fit the data, we additionally perform model selection. We dedicate one fold as the validation set, and use the remaining folds to find the best parameter values of both CUR ${ }^{2}$ LED and TILDE. Table 2 summarizes the results and reports the selected choice of parameter, together with the performance on the validation set. These results are consistent with the ones in Table 1: $\mathcal{C}$-representation improves the performance in the majority of cases, and the selected parameters correspond to the best performing ones in Table 1 .

Q5. Table 1 shows that CUR ${ }^{2}$ LED substantially outperforms MRC on all datasets, achieving better performance on all datasets except the IMDB. Moreover, MRC rarely shows benefit over the original data representation, with an exception on the Hepatitis dataset. Considering the model complexity, the models learned on MRC-induced representation are substantially more complex than the ones learned on $\mathcal{C}$ representations. Table 3 summarize the number of clusters created by CUR $^{2}$ LED and MRC. MRC creates substantially more clusters than CUR ${ }^{2}$ LED . Because of this, the found clusters contain only a few objects which makes it difficult to generalize well, and increases the model complexity. The number of clusters found by CUR ${ }^{2}$ LED is relatively high, because it finds a representation of data suitable for many classification tasks over the same datasets. Thus, most of the features are redundant for one specific task, but clearly contain better information as the models learned on them perform better and
Table 3: Vocabulary sizes. M indicates MRC, while $\mathrm{S}$ and $\mathrm{H}$ indicate CUR $^{2}$ LED representations with spectral and hierarchical clustering, respectively. Vocabulary sizes obtained with merging and combination similarities were similar, so only the one for merging is reported.

\begin{tabular}{ccccccc}
\hline Setup & UW & Muta & WebKB & Terror & IMDB & Hepa \\
\hline Original & 10 & 12 & 775 & 107 & 5 & 22 \\
\hline S, $\alpha=0.01$ & 109 & 53 & 65 & 30 & 75 & 85 \\
S, $\alpha=0.05$ & 87 & 37 & 63 & 26 & 69 & 66 \\
S, $\alpha=0.1$ & 72 & 31 & 57 & 24 & 59 & 28 \\
S, silhouette & 93 & 17 & 59 & 37 & 74 & 79 \\
\hline $\mathbf{H}, \alpha=0.01$ & 93 & 38 & 64 & 25 & 69 & 62 \\
$\mathbf{H}, \alpha=0.05$ & 85 & 34 & 64 & 20 & 65 & 50 \\
$\mathbf{H}, \alpha=0.1$ & 68 & 22 & 58 & 18 & 55 & 46 \\
$\mathbf{H}$, silhouette & 85 & 20 & 55 & 43 & 64 & 61 \\
\hline $\mathbf{M}, \lambda=-1$ & 183 & 535 & 817 & 318 & 49 & 655 \\
$\mathbf{M}, \lambda=-5$ & 140 & 346 & 331 & 116 & 38 & 297 \\
$\mathbf{M}, \lambda=-10$ & 49 & 224 & 219 & 91 & 18 & 120 \\
\hline
\end{tabular}

have lower complexity.

The computational complexity of CUR ${ }^{2}$ LED is impossible to state in general, as it depends on the choice of (and is dominated by) the clustering algorithm. Performing a 5-fold cross validation on a single CPU took approximately 5 minutes for the IMDB and UWCSE datasets, 24 hours for the Terrorists dataset and approximately a week for the remaining datasets. Though expensive, latent representation has to be created only once and can be reused for many tasks with the same dataset. Moreover, CUR ${ }^{2}$ LED is easily parallelizable which can substantially improve its efficiency.

\section{Conclusion}

This work introduces CUR ${ }^{2}$ LED - a clustering-based framework for unsupervised representation learning with relational data, which describes both instances and relationships between them. Viewing relational data as hypergraph, CUR ${ }^{2}$ LED learns new features by clustering both instances and their relationships. i.e., vertices and hyperedges in the corresponding hypergraph. To support such procedure, we introduce a general hyperedges clustering framework based on similarity of vertices participating in the hyperedge. A distinct feature of CUR ${ }^{2}$ LED is the way it uses the ambiguity of similarity within relational data, i.e., whether two relational objects are similar due to their features of relationships, to generate distributed representation of data. We design several experiments to verify the usefulness of latent representation generated by CUR ${ }^{2}$ LED . The results show that the latent representations created by CUR $^{2}$ LED provide a better representation of data that results in models of lower complexity and better performance. In future work, we will extend CUR ${ }^{2}$ LED towards semi-supervised settings, and investigate alternative ways for learning a distributed representations directly from data.

\section{Acknowledgments}

This research is supported by Research Fund KU Leuven (GOA/13/010). 


\section{References}

[Arbelaitz et al., 2013] Olatz Arbelaitz, Ibai Gurrutxaga, Javier Muguerza, JesúS M. PéRez, and IñIgo Perona. An extensive comparative study of cluster validity indices. Pattern Recogn., 46(1):243-256, January 2013.

[Bengio et al., 2007] Yoshua Bengio, Pascal Lamblin, Dan Popovici, and Hugo Larochelle. Greedy layer-wise training of deep networks. In NIPS. MIT Press, 2007.

[Bengio et al., 2013] Yoshua Bengio, Aaron Courville, and Pascal Vincent. Representation learning: A review and new perspectives. IEEE Trans. Pattern Anal. Mach. Intell., 35(8):1798-1828, August 2013.

[Bengio, 2009] Yoshua Bengio. Learning deep architectures for ai. Found. Trends Mach. Learn., 2(1):1-127, January 2009.

[Blockeel and De Raedt, 1998] Hendrik Blockeel and Luc De Raedt. Top-down induction of first-order logical decision trees. Artificial Intelligence, 101(12):285 - 297, 1998.

[Bordes et al., 2011] Antoine Bordes, Jason Weston, Ronan Collobert, and Yoshua Bengio. Learning structured embeddings of knowledge bases. In Proceedings of the TwentyFifth AAAI Conference on Artificial Intelligence, AAAI'11, pages 301-306. AAAI Press, 2011.

[Bordes et al., 2013] Antoine Bordes, Nicolas Usunier, Alberto Garcia-Durán, Jason Weston, and Oksana Yakhnenko. Translating embeddings for modeling multi-relational data. In Proceedings of the 26th International Conference on Neural Information Processing Systems, NIPS'13, pages 2787-2795, USA, 2013. Curran Associates Inc.

[Coates et al., 2011] A. Coates, H. Lee, and A.Y. Ng. An analysis of single-layer networks in unsupervised feature learning. In Proceedings of the Fourteenth International Conference on Artificial Intelligence and Statistics, volume 15 of JMLR Workshop and Conference Proceedings, pages 215-223. JMLR W\&CP, 2011.

[Craven and Slattery, 2001] Mark Craven and Seán Slattery. Relational learning with statistical predicate invention: Better models for hypertext. Mach. Learn., 43(1-2):97-119, April 2001.

[Dumančić and Blockeel, 2017] Sebastijan Dumančić and Hendrik Blockeel. An expressive dissimilarity measure for relational clustering using neighbourhood trees. $M a$ chine learning, 2017. To appear.

[Fortunato, 2010] Santo Fortunato. Community detection in graphs. Physics Reports, 486(3-5):75 - 174, 2010.

[Hinton and Salakhutdinov, 2006] G. E. Hinton and R. R. Salakhutdinov. Reducing the dimensionality of data with neural networks. Science, 313(5786):504-507, 2006.

[Karypis and Kumar, 1998] George Karypis and Vipin Kumar. A fast and high quality multilevel scheme for partitioning irregular graphs. SIAM J. Sci. Comput., 20(1):359-392, December 1998.
[Kok and Domingos, 2007] Stanley Kok and Pedro Domingos. Statistical predicate invention. In Proceedings of the 24th International Conference on Machine Learning, ICML '07, pages 433-440, New York, NY, USA, 2007. ACM.

[Kok and Domingos, 2008] Stanley Kok and Pedro Domingos. Extracting semantic networks from text via relational clustering. In Proceedings of the 2008 European Conference on Machine Learning and Knowledge Discovery in Databases - Part I, ECML PKDD '08, pages 624-639, Berlin, Heidelberg, 2008. Springer-Verlag.

[Kramer, 1995] Stefan Kramer. Predicate Invention: A Comprehensive View. Technical report, 1995.

[Nickel et al., 2016] Maximilian Nickel, Kevin Murphy, Volker Tresp, and Evgeniy Gabrilovich. A review of relational machine learning for knowledge graphs. Proceedings of the IEEE, 104(1):11-33, 2016.

[Niepert et al., 2016] Mathias Niepert, Mohamed Ahmed, and Konstantin Kutzkov. Learning convolutional neural networks for graphs. In Proceedings of the 33nd International Conference on Machine Learning, ICML 2016, New York City, NY, USA, June 19-24, 2016, pages 2014-2023, 2016.

[Niepert, 2016] Mathias Niepert. Discriminative gaifman models. In Advances in Neural Information Processing Systems 29: Annual Conference on Neural Information Processing Systems 2016, December 5-10, 2016, Barcelona, Spain, pages 3405-3413, 2016.

[Popescul and Ungar, 2004] Alexandrin Popescul and Lyle H. Ungar. Cluster-based concept invention for statistical relational learning. In Proceedings of the Tenth ACM SIGKDD International Conference on Knowledge Discovery and Data Mining, KDD '04, pages 665-670, New York, NY, USA, 2004. ACM.

[Ranzato et al., 2007] Marc'Aurelio Ranzato, Y-Lan Boureau, and Yann LeCun. Sparse feature learning for deep belief networks. In NIPS, pages 1185-1192. Curran Associates, Inc., 2007.

[Richardson and Domingos, 2006] Matthew Richardson and Pedro Domingos. Markov logic networks. Mach. Learn., 62(1-2):107-136, February 2006.

[Rousseeuw, 1987] Peter Rousseeuw. Silhouettes: A graphical aid to the interpretation and validation of cluster analysis. J. Comput. Appl. Math., 20(1):53-65, November 1987.

[Vendramin et al., 2010] Lucas Vendramin, Ricardo J. G. B. Campello, and Eduardo R. Hruschka. Relative clustering validity criteria: A comparative overview. Statistical Analysis and Data Mining, 3(4):209-235, 2010. 